\title{
A simple recurrence for covers of the sphere with branch points of arbitrary ramification
}

\author{
I. P. Goulden* and Luis G. Serrano ${ }^{\dagger}$ \\ University of Waterloo \\ Department of Combinatorics \& Optimization \\ Waterloo, Ontario N2L 3G1, Canada
}

November 8, 2018

\begin{abstract}
The problem of counting ramified covers of a Riemann surface up to homeomorphism was proposed by Hurwitz in the late 1800's. This problem translates combinatorially into factoring a permutation of specified cycle type, with certain conditions on the cycle types of the factors, such as minimality and transitivity.

Goulden and Jackson have given a proof for the number of minimal, transitive factorizations of a permutation into transpositions. This proof involves a partial differential equation for the generating series, called the Join-Cut equation. Recently, Bousquet-Mélou and Schaeffer have found the number of minimal, transitive factorizations of a permutation into arbitrary unspecified factors. This was proved by a purely combinatorial argument, based on a direct bijection between factorizations and certain objects called $m$-Eulerian trees.

In this paper, we give a simple partial differential equation for Bousquet-Mélou and Schaeffer's generating series, and for Goulden and Jackson's generating series, as well as a new proof of the result by Bousquet-Mélou and Schaeffer. We apply algebraic methods based on Lagrange's theorem, and combinatorial methods based on a new use of Bousquet-Mélou and Schaeffer's $m$-Eulerian trees.
\end{abstract}

\section{Introduction}

\subsection{Background and notation}

The subject of this paper is a mathematical problem that has attracted significant attention in the last decade, with contributions from researchers in algebraic combinatorics, algebraic geometry, and mathematical physics. Its origins are in the work of Hurwitz [5], dating from the 1890's.

First, for some notation. A partition is a weakly ordered list of positive integers $\lambda=\left(\lambda_{1}, \ldots, \lambda_{k}\right)$, where $\lambda_{1} \geq \ldots \geq \lambda_{k}$. The integers $\lambda_{1}, \ldots, \lambda_{k}$ are called the parts of the partition $\lambda$, and we denote the number of parts by $l(\lambda)=k$. If $\lambda_{1}+\ldots+\lambda_{k}=n$, then $\lambda$ is a partition of $n$, and we write $\lambda \vdash n$. In the symmetric group $S_{n}$ on $\{1, \ldots, n\}$, let $\mathcal{C}_{\lambda}$ be the conjugacy class consisting of all permutations whose disjoint cycle lengths are specified by the parts of the partition $\lambda$. If $\lambda$ has $d_{i}$ parts equal to $i$, for $i \geq 1$, then it is well known that $\left|\mathcal{C}_{\lambda}\right|=n ! / \prod_{i \geq 1} i^{d_{i}} d_{i} !$

In this paper, we consider branched covers of the sphere by an $n$-sheeted Riemann surface of genus $g$. Suppose that the branch points are $P_{0}, P_{1}, \ldots, P_{k}$, with ramification at $P_{i}$ specified by $\pi_{i} \in P_{i}$, for $i=$ $0, \ldots, k$. (This means that if one walks in a small neighbourhood, counterclockwise, around $P_{i}$, starting at sheet $j$, then one ends at sheet $\pi_{i}(j)$.) The monodromy condition states that, for consistency, we must have

$$
\pi_{1} \ldots \pi_{k}=\pi
$$

\footnotetext{
* Supported by a Discovery Grant from NSERC. E-mail ipgoulden@uwaterloo.ca

${ }^{\dagger}$ Research supported by a Postgraduate Scholarship from NSERC. E-mail lgserrano@uwaterloo.ca
} 
where $\pi=\pi_{0}^{-1}$. For the cover to be connected, one must be able to move from sheet $j$ to sheet $m$, by walking around suitable branch points, for any choices of $j$ and $m$. This means that the group generated by $\pi_{1}, \ldots, \pi_{k}$ must be a transitive subgroup of $S_{n}$. In this case, we call the factorization of $\pi$ in (10) a transitive factorization. Finally, if $\pi_{i} \in \mathcal{C}_{\alpha_{i}}, i=0, \ldots, n$, then we say that the ramification-type of $P_{i}$ is $\alpha_{i}$, and the Riemann-Hurwitz formula then gives

$$
\sum_{i=0}^{k}\left(n-l\left(\alpha_{i}\right)\right)=2 n-2+2 g .
$$

In the case $g=0$, we call the factorization a minimal transitive factorization. Hurwitz [5] proved that the ordered $\pi_{i}$ 's specify a unique cover up to homeomorphism, so we can count (homeomorphically) distinct branched covers with specified ramification by counting transitive factorizations. A branch point whose ramification is a transposition is called a simple branch point.

In genus 0 , there are two explicit, general results known. For the first result, let $H_{\alpha}$ be the number of distinct covers of the sphere by an $n$-sheeted Riemann surface of genus 0 , with fixed ramification of type $\alpha$ at $P_{0}$, and all other branch points are simple. Then Goulden and Jackson [4] (see also Hurwitz [6]) have proved that

$$
H_{\alpha}=n^{l(\alpha)-3}(n+l(\alpha)-2) ! \prod_{i \geq 1}\left(\frac{i^{i}}{(i-1) !}\right)^{d_{i}},
$$

where $\alpha$ has $d_{i}$ parts equal to $i, i \geq 1$. In this case, the Riemann-Hurwitz formula (2) gives the number of simple branch points as $n+l(\alpha)-2$.

For the second explicit result, let $G_{\alpha}(m)$ be the number of distinct covers of the sphere by an $n$-sheeted Riemann surface of genus 0 , with fixed ramification of type $\alpha$ at $P_{0}$, and $m$ other branch points with arbitrary ramification. Then Bousquet-Mélou and Schaeffer [1] have proved that

$$
G_{\alpha}(m)=m \frac{\{(m-1) n-1\} !}{\{(m-1) n-l(\alpha)+2\} !} \prod_{i \geq 1}\left\{i\left(\begin{array}{c}
m i-1 \\
i
\end{array}\right)\right\}^{d_{i}}
$$

where $\alpha$ has $d_{i}$ parts equal to $i, i \geq 1$. In this case, the Riemann-Hurwitz formula (2) gives the total number of cycles in the arbitrary factors as $c(\alpha)=(m-1) n-l(\alpha)+2$.

The following version of Lagrange's Theorem, as it appears in $[3]$, is used in this paper. We write $[A] B$ to mean "the coefficient of $A$ in $B$ ".

Theorem 1 (Lagrange) Let $\phi(\lambda) \in R[[\lambda]]_{1}$. Then there exists a unique formal power series $w(t) \in R[[t]]_{0}$ such that $w=t \phi(w)$. Moreover, if $f(\lambda) \in R((\lambda))$, then for $n \neq 0$,

$$
\left[t^{n}\right] f(w)=\frac{1}{n}\left[\lambda^{n-1}\right] f^{\prime}(\lambda) \phi^{n}(\lambda) .
$$

\subsection{Outline}

In Section 2] we apply Lagrange's Theorem to prove that a generating series for Bousquet-Mélou and Schaeffer's [1] numbers $G_{\alpha}(m)$, given in (4), satisfies a simple quadratic partial differential equation. This equation is given in Theorem [3] In Section [3 we give a combinatorial proof of Theorem [3 and the associated Lagrangian equations, using a minor adaptation of the combinatorial objects introduced by Bousquet-Mélou and Schaeffer [1] in their proof of (4). In Section [4 we state, as Theorem [6] a simple quadratric partial differential equation for a generating series for the numbers $H_{\alpha}$, given in (3). We know of no direct combinatorial or geometric proof of either Theorem [3 or Theorem [6] despite the apparent simplicity of these equations.

Moreover, the numbers $H_{\alpha}$ satisfy a well-known partial differential equation called the Join-Cut equation. (This equation was used in [4 to obtain (3).) The Join-Cut equation has a simple combinatorial proof, by analyzing the transpositions in a corresponding factorization, and a simple geometric proof, by analyzing branch points. The Join-Cut equation also extends in a straightforward way to the case of arbitrary genus. In arbitrary genus, the corresponding numbers (in which one fixed branch point has arbitrary ramification, 
and all others are simple) are given by the ELSV formula 2], as a Hodge integral. However, we know of no simple combinatorial or geometric proof of Theorem [ 6

For the numbers $G_{\alpha}(m)$, we know of no associated results analogous to those described above for the numbers $H_{\alpha}$. We speculate that analogues of some of these associated results exist, and are particularly interested in extensions to higher genus.

\section{Branch points of arbitrary ramification}

In this section we consider the following generating series for the numbers $G_{\alpha}(m)$ given in (4):

$$
G \equiv G\left(z, u, x, p_{1}, p_{2}, \ldots\right)=\sum_{n \geq 1} \sum_{\alpha \vdash n} G_{\alpha}(m) \frac{z^{n}}{n !}\left|\mathcal{C}_{\alpha}\right| u^{l(\alpha)} p_{\alpha} x^{(m-1) n-l(\alpha)+2} .
$$

Here $z$ is an exponential indeterminate marking the elements in $S_{n}, u$ is an ordinary indeterminate marking the cycles in $\pi$, and $x$ is an ordinary indeterminate marking the total number of cycles of the factors $\pi_{1}, \ldots, \pi_{m}$, which by the minimality condition is $c(\alpha)=(m-1) n-l(\alpha)+2$.

For fixed $m \geq 1$, let $A(t)=\sum_{i \geq 1}\left(\begin{array}{c}m i-1 \\ i\end{array}\right) u p_{i} t^{i}$, and $w \equiv w\left(z, u, x, p_{1}, p_{2}, \ldots\right)$ be the unique formal power series satisfying

$$
w=z(x+A(w))^{m-1} .
$$

In the following result, we use (4) to express two partial derivatives of $G\left(z, u, p_{1}, p_{2}, \ldots\right)$ in terms of $A(w)$. Of course, we can determine $w$ and $A(w)$ by Lagrange's Theorem.

Theorem 2 For $G$ given by (5), and $w$ given by (6), we have

$$
\left(z \frac{\partial}{\partial z}\right)\left((m-1) z \frac{\partial}{\partial z}+1\right) G=\frac{m}{m-1} x A(w)+\frac{m}{2(m-1)} A(w)^{2},
$$

and

$$
\left(z \frac{\partial}{\partial z}\right)\left(\frac{\partial}{\partial x}\right) G=\frac{m}{m-1} A(w)
$$

Proof. From (44), and the fact that $\left|\mathcal{C}_{\alpha}\right|=n ! / \prod_{i \geq 1} i^{d_{i}} d_{i}$ !, we obtain

$$
G=\sum_{n \geq 1} z^{n} \sum_{\alpha \vdash n} m \frac{\{(m-1) n-1\} !}{\{(m-1) n-l(\alpha)+2\} !} x^{(m-1) n-l(\alpha)+2} \prod_{i \geq 1}\left(\begin{array}{c}
m i-1 \\
i
\end{array}\right)^{d_{i}} \frac{p_{i}^{d_{i}}}{d_{i} !} u^{d_{i}},
$$

where $\alpha$ has $d_{i}$ parts equal to $i$, for $i \geq 1$. Thus, we have, for $n \geq 1$,

$$
\begin{aligned}
{\left[z^{n}\right] G } & =\sum_{k \geq 1} m \frac{\{(m-1) n-1\} !}{\{(m-1) n-k+2\} !} \sum_{\begin{array}{c}
d_{1}+2 d_{2}+\cdots=n \\
d_{1}+d_{2}+\cdots=k
\end{array}} x^{(m-1) n-k+2} \prod_{i \geq 1}\left(\begin{array}{c}
m i-1 \\
i
\end{array}\right)^{d_{i}} \frac{p_{i}^{d_{i}}}{d_{i} !} u^{d_{i}} \\
& =\sum_{k \geq 1} m \frac{\{(m-1) n-1\} !}{\{(m-1) n-k+2\} !}\left[\lambda^{n}\right] \frac{A(\lambda)^{k}}{k !} x^{(m-1) n-k+2},
\end{aligned}
$$


from the multinomial theorem. Thus, we obtain

$$
\begin{aligned}
{\left[z^{n}\right] z \frac{\partial}{\partial z}\left((m-1) z \frac{\partial}{\partial z}+1\right) G } & =\sum_{k \geq 1} n\{(m-1) n+1\} m \frac{\{(m-1) n-1\} !}{\{(m-1) n-k+2\} !}\left[\lambda^{n}\right] \frac{A(\lambda)^{k}}{k !} x^{(m-1) n-k+2} \\
& =\sum_{k \geq 1} \frac{m}{(m-1)\{(m-1) n+2\}}\left(\begin{array}{c}
(m-1) n+2 \\
k
\end{array}\right)\left[\lambda^{n}\right] A(\lambda)^{k} x^{(m-1) n-k+2} \\
& =\frac{m}{(m-1)\{(m-1) n+2\}}\left[\lambda^{n}\right](x+A(\lambda))^{(m-1) n+2} \\
& =\frac{m}{(m-1)\{(m-1) n+2\}} \frac{1}{n}\left[\lambda^{n-1}\right] \frac{d}{d \lambda}(x+A(\lambda))^{(m-1) n+2} \\
& =\frac{m}{m-1} \frac{1}{n}\left[\lambda^{n-1}\right] A^{\prime}(\lambda)(x+A(\lambda))^{(m-1) n+1} \\
& =\frac{m}{m-1}\left[z^{n}\right]\left(x A(w)+\frac{1}{2} A(w)^{2}\right)
\end{aligned}
$$

from Theorem 11 and (7) follows, since both $G$ and $A(w)$ are equal to 0 at $z=0$.

Similarly, we obtain

$$
\begin{aligned}
{\left[z^{n}\right] z \frac{\partial}{\partial z}\left(\frac{\partial}{\partial x}\right) G } & =\sum_{k \geq 1} n\{(m-1) n-k+2\} m \frac{\{(m-1) n-1\} !}{\{(m-1) n-k+2\} !}\left[\lambda^{n}\right] \frac{A(\lambda)^{k}}{k !} x^{(m-1) n-k+1} \\
& =\sum_{k \geq 1} \frac{m}{(m-1)\{(m-1) n+1\}}\left(\begin{array}{c}
(m-1) n+1 \\
k
\end{array}\right)\left[\lambda^{n}\right] A(\lambda)^{k} x^{(m-1) n-k+1} \\
& =\frac{m}{(m-1)\{(m-1) n+1\}}\left[\lambda^{n}\right](x+A(\lambda))^{(m-1) n+1} \\
& =\frac{m}{(m-1)\{(m-1) n+1\}} \frac{1}{n}\left[\lambda^{n-1}\right] \frac{d}{d \lambda}(x+A(\lambda))^{(m-1) n+1} \\
& =\frac{m}{m-1} \frac{1}{n}\left[\lambda^{n-1}\right] A^{\prime}(\lambda)(x+A(\lambda))^{(m-1) n} \\
& =\frac{m}{m-1}\left[z^{n}\right] A(w),
\end{aligned}
$$

from Theorem [1, and (8) follows.

Note that since

$$
\left(x \frac{\partial}{\partial x}\right) G=\left((m-1) z \frac{\partial}{\partial z}-u \frac{\partial}{\partial u}+2\right) G,
$$

then we can eliminate $A(w)$ between equations (7) and (8), to obtain a quadratic partial differential equation for $G$, given in the following result.

Theorem 3 The generating series $G$ satisfies the partial differential equation

$$
2 m z \frac{\partial}{\partial z}\left(u \frac{\partial}{\partial u}-1\right) G=(m-1)\left(z \frac{\partial}{\partial z} \frac{\partial}{\partial x} G\right)^{2} .
$$

\section{$3 m$-Eulerian trees}

In this section we will provide a combinatorial interpretation for the partial differential equations in Section 2. With the purpose of interpreting (10), we will make use of certain objects called $m$-Eulerian trees, defined by Bousquet-Mélou and Schaeffer in [1. A leaf in a tree is a vertex of degree 1, and an inner vertex is one that is not a leaf. The inner degree of a vertex is the number of inner neighbours. An m-Eulerian tree is a (properly) coloured tree where the colours of the vertices are black and white, and has the following two properties: 
- Every inner black vertex has total degree $m$ and inner degree 1 or 2 ,

- Every inner white vertex has total degree $m i$ for some $i \geq 1$, and exactly $i-1$ neighbours of inner degree 1.

Now, it is straightforward to prove by a counting argument that the number of black leaves is $m$ greater than the number of white leaves in an $m$-Eulerian tree. Bousquet-Mélou and Schaeffer [1] have proved that there is a unique way of inserting edges between white leaves and black leaves in such a way that the outer face in the resulting planar map contains the $m$ unpaired black leaves. An $m$-Eulerian tree is said to be planted if a black leaf is specified as a root vertex. Furthermore, it is said to be balanced if the root is precisely one of these $m$ black unpaired leaves. Note that in this paper, we allow an $m$-Eulerian tree that is not planted (notice the slight difference between this definition and that in 1]).

If $\alpha \vdash n$ has $d_{i}$ parts of size $i, i \geq 1$, an $m$-Eulerian tree of type $\alpha$ is defined as having $n-1$ black inner vertices, $l(\alpha)$ white inner vertices, $d_{i}$ of which have total degree $m i$, and $c(\alpha)$ black leaves. An example can be seen in Figure 1 where inner vertices are drawn as squares, and leaves are drawn as circles.

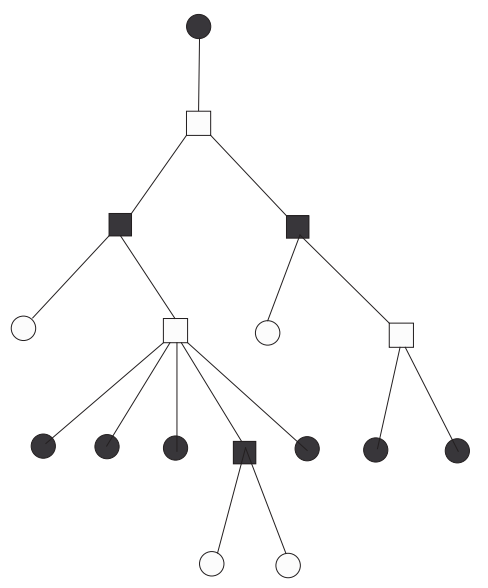

Figure 1: A Balanced m-Eulerian Tree

Bousquet-Mélou and Schaeffer have proved (4) by finding a bijection between minimal transitive factorizations of a permutation of type $\alpha$ into $m$ factors, and balanced $m$-Eulerian trees of type $\alpha$. In this bijection, the element 1 becomes a new separate black vertex of degree $m$, that is inserted into the outer face of the map described above, and joined to the $m$ unpaired black leaves. The remaining elements of $\{2, \ldots, n\}$ correspond to the inner black vertices, the cycles of $\alpha$ to the inner white vertices, and the cycles of the $m$ factors correspond to the black leaves. Furthermore, to turn the $m$-Eulerian tree into a balanced tree, one can plant it in $m$ ways. Therefore, if $T_{\alpha}(m)$ is the number of $m$-Eulerian trees of type $\alpha$, then we have

$$
m T_{\alpha}(m)=\frac{\left|\mathcal{C}_{\alpha}\right|}{(n-1) !} G_{\alpha}(m) .
$$

Thus, by defining the generating series $T$ as

$$
T \equiv T\left(z, u, x, p_{1}, p_{2}, \ldots\right)=\sum_{n \geq 1} \sum_{\alpha \vdash n} T_{\alpha}(m) z^{n-1} u^{l(\alpha)} p_{\alpha} x^{(m-1) n-l(\alpha)+2},
$$

where $z$ is an ordinary marker for the inner black vertices, $u$ an ordinary marker for the inner white vertices, and $x$ an ordinary marker for the black leaves, we obtain

$$
m T=\frac{\partial}{\partial z} G
$$

Applying (11), equation (10) for $G$ becomes the following equation for $\mathrm{T}$ :

$$
2\left(u \frac{\partial}{\partial u}-1\right) T=(m-1) z\left(\frac{\partial}{\partial x} T\right)^{2} .
$$


Using $m$-Eulerian trees, we have the following combinatorial proof of (12). Consider two $m$-Eulerian trees (possibly identical). Root each one at a black leaf (not necessarily an unpaired one). Now, identify these two roots, to become a new inner black vertex of degree 2, thus obtaining the generating series $z\left(\frac{\partial}{\partial x} T\right)^{2}$. Note that the tree obtained has the properties of an $m$-Eulerian tree at every vertex except for the new one, and all we need to do to satisfy the conditions of an $m$-Eulerian tree is to attach $m-2$ leaves to this new vertex. This can be done in $m-1$ ways, since we simply decide how many leaves go on each side of the vertex, thus giving the right hand side of (12).

For the left hand side of (12), note that this process determines a new $m$-Eulerian tree, with a selected inner black vertex of inner degree 2. In order to count the number of inner black vertices of inner degree 2, root the $m$-Eulerian tree at any black leaf. Then every inner white vertex, except for the one adjacent to the root, can be paired up uniquely with the inner black vertex of inner degree 2 that immediately follows it in the unique path from the white vertex to the root. Therefore there are $l(\alpha)-1$ of these black vertices. Thus the generating series for selecting one of these is $\left(u \frac{\partial}{\partial u}-1\right) T$. Note that in this bijection every Eulerian tree is constructed twice, which accounts for the factor of 2 on the left hand side of (12). This process, which is clearly bijective, is illustrated in Figure 2

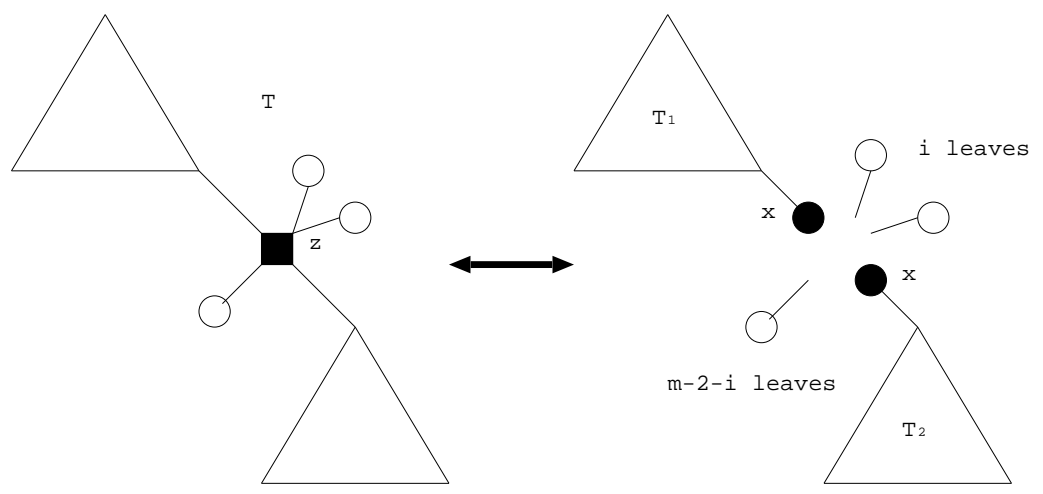

Figure 2: Cutting the Tree

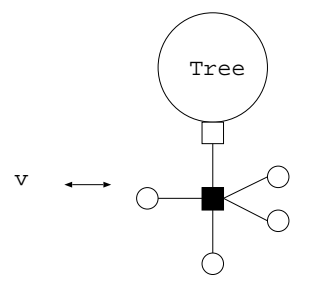

Figure 3: A pseudo-Eulerian Tree

We can also interpret the Lagrangian equations in Section 2 In order to do so, we define a pseudoEulerian tree. This tree is constructed by removing the root of a planted $m$-Eulerian tree (note that it does not need to be balanced), and adding an inner black vertex in its place, with $m-1$ new white leaves emanating from it. As a final step, we root this new tree at one of these $m-1$ white leaves. A pseudoEulerian tree is illustrated in Figure 3 For $\alpha \vdash n$, let $v_{\alpha}$ be the number of pseudo-Eulerian trees with $n$ inner black vertices, $l(\alpha)$ inner white vertices, and $(m-1) n-l(\alpha)+1$ black leaves, and define $v$ as the generating series

$$
v \equiv v\left(z, u, x, p_{1}, p_{2}, \ldots\right)=\sum_{\alpha \vdash n} v_{\alpha} z^{n} u^{l(\alpha)} p_{\alpha} x^{(m-1) n-l(\alpha)+1} .
$$


Then, by the construction described above,

$$
v=(m-1) z \frac{\partial}{\partial x} T .
$$

Note that by (8), (11), and (13), we can conclude that

$$
v=A(w) .
$$

Now, define a list as a list of $m$ objects, the first of which is an inner black vertex, and the remaining $m-1$ are a choice of either a black leaf, or a pseudo-Eulerian tree, as seen on Figure 4 Note that this implies that the generating series for lists, where the variables $z, x, u$, and $p_{i}$ mark the same objects as before, is $z(x+v)^{m-1}$, which by (6) and (14) is equal to $w$.

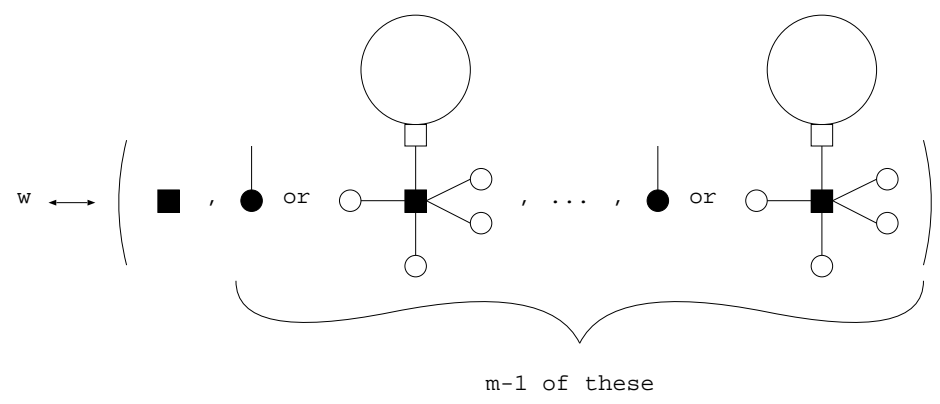

Figure 4: A list
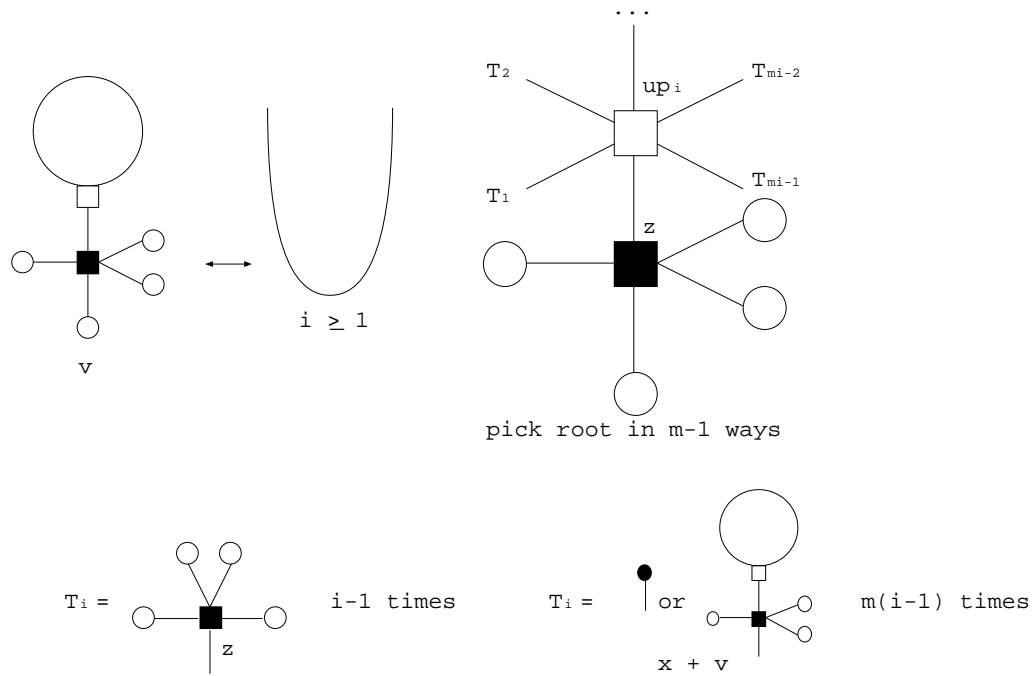

Figure 5: Decomposition of pseudo-Eulerian trees

In order to interpret (14), we decompose a pseudo-Eulerian tree. Note that a pseudo-Eulerian planted tree is planted at a white leaf, which is selected in $m-1$ ways, and adjacent to a black inner vertex of inner degree 1 , marked by $z$. This inner vertex is adjacent to exactly one inner white vertex of degree $m i$, for some $i \geq 1$, marked by $u p_{i}$ Now, for the remaining $m i-1$ edges that emanate from this white vertex, $i-1$ of them must be adjacent to an inner black vertex of inner degree 1, and their positions can be chosen in $\left(\begin{array}{c}m i-1 \\ i-1\end{array}\right)$ ways. Each one of them is marked by $z$. So we have an ordered set of $i$ copies of the variable $z$, starting from the black vertex adjacent to the root, and going clockwise around the white vertex specified above. Now, on the remaining $m i-i$ edges adjacent to this white vertex, we must attach either a black leaf, or a pseudo-Eulerian tree, together giving a factor of $x+v$. Again, taking them clockwise, we have 
an ordered list of $(m-1) i$ objects, each one being a choice of a black leaf or a pseudo-Eulerian tree. Note that all these, together with the $m$ inner black vertices of inner degree one above, can be arranged into an ordered set of $i$ lists, in the canonical way, each marked by $w$. The equation obtained by this reasoning is $v=(m-1) \sum_{i \geq 1}\left(\begin{array}{c}m i-1 \\ i-1\end{array}\right) u p_{i} w^{i}=A(w)$. This process, which is clearly reversible, can be seen more clearly in Figure 5

This concludes the combinatorial interpretation of the partial differential equations in Section 2 Moreover, by Theorem 1 (6), and (13), one can recover (4), thus finding a different proof of the result in [1].

\section{Simple branch points}

In this section we consider the following generating series for the numbers $H_{\alpha}$ given in (3):

$$
H \equiv H\left(z, p_{1}, p_{2}, \ldots\right)=\sum_{n \geq 1} \sum_{\alpha \vdash n} H_{\alpha} \frac{z^{n}}{n !} \frac{\left|\mathcal{C}_{\alpha}\right|}{(n+l(\alpha)-2) !} p_{\alpha} .
$$

Let $B(t)=\sum_{i \geq 1} \frac{i^{i}}{i !} p_{i} t^{i}$, and let $s \equiv s\left(z, p_{1}, p_{2}, \ldots\right)$ be the unique formal power series satisfying

$$
s=z \exp B(s) .
$$

The following result, from [4, expresses three partial derivatives of $H\left(z, p_{1}, p_{2}, \ldots\right)$ in terms of $s$ and $B(s)$.

Theorem 4 For $H$ given by (15), and s given by (16), we have

$$
\begin{gathered}
\left(z \frac{\partial}{\partial z}\right)^{2} H=B(s), \\
z \frac{\partial}{\partial z} H=\sum_{i \geq 1} \frac{i^{i-1}}{i !} p_{i} s^{i}-\frac{1}{2} B(s)^{2},
\end{gathered}
$$

and for $k \geq 1$,

$$
z \frac{\partial}{\partial z} \frac{\partial}{\partial p_{k}} H=\frac{k^{k-1}}{k !} s^{k}
$$

The following result gives a simple quadratic partial differential equation for $H$, that follows immediately from Theorem 4. This result has been obtained by Goulden and Jackson (private communication).

Theorem 5 The generating series $H$ satisfies the partial differential equation

$$
z \frac{\partial}{\partial z} H=z \frac{\partial}{\partial z} \sum_{k \geq 1} p_{k} \frac{\partial}{\partial p_{k}} H-\frac{1}{2}\left(\left(z \frac{\partial}{\partial z}\right)^{2} H\right)^{2}
$$

Proof. Substitute (17) and (19), after adding over all $k$, into (18).

This partial differential equation can be transformed in a straightforward way to have a simpler form, by considering the series

$$
\widehat{H}=\sum_{n \geq 1} \sum_{\alpha \vdash n} H_{\alpha} \frac{z^{n}}{n !} \frac{\left|\mathcal{C}_{\alpha}\right|}{(n-l(\alpha)+2) !} p_{\alpha} u^{l(\alpha)} .
$$

Note that, in the generating series $\widehat{H}, z$ is an exponential indeterminate marking the elements in $S_{n}$, u is an ordinary indeterminate marking the cycles in $\pi$, and $p_{i}$ is an ordinary indeterminate marking the cycles of length $i$ in $\pi$.

Theorem 6 The generating series $\widehat{H}$ satisfies the partial differential equation

$$
z \frac{\partial}{\partial z} \widehat{H}=z \frac{\partial}{\partial z} u \frac{\partial}{\partial u} \widehat{H}-\frac{1}{2}\left(\left(z \frac{\partial}{\partial z}\right)^{2} \widehat{H}\right)^{2}
$$


Proof. The result follows from Theorem 5 and the fact that $\left(u \frac{\partial}{\partial u}\right) \widehat{H}=\left(\sum_{k \geq 1} p_{k} \frac{\partial}{\partial p_{k}}\right) \widehat{H}$.

Note that in this context, $u$ keeps track of the cycles in $\pi$. The apparent simplicity of Theorem 6 . especially as it involves only the two variables $z$ and $u$, suggests that there may be a simple combinatorial interpretation. We have looked for such a combinatorial interpretation, but have been unable to find one.

\section{References}

[1] M. Bousquet-Mélou, G. Schaeffer, Enumeration of Planar Constellations, Adv. Appl. Math. 24 (2000), 337-368.

[2] T. Ekedahl, S. Lando, M. Shapiro, A. Vainshtein, Hurwitz Numbers and Intersections on Moduli Spaces of Curves, Invent. Math. 146 (2001), 297-327.

[3] I.P. Goulden, D.M. Jackson, “Combinatorial Enumeration”, John Wiley \& Sons, New York, 1983 (Dover Reprint, 2004).

[4] I.P. Goulden, D.M. Jackson, Transitive Factorizations into Transpositions and Holomorphic Mappings on the Sphere, Proc. Amer. Math. Soc. 125 (1997), 51-60.

[5] A. Hurwitz, Über Riemann'sche Flächen mit gegebenen Verzweignungspunkten, Math. Ann. 39 (1891), 1-60.

[6] A. Hurwitz, Über die Anzahl der Riemann'schen Flächen mit gegebenen Verzweignungspunkten, Math. Ann. 55 (1902), 53-66. 